\title{
Magneto-thermo-visco-elastic waves in an initially stressed conducting layer
}

\author{
AMIT KUMAR RAKSHIT ${ }^{\prime}$ and P R SENGUPTA ${ }^{2}$ \\ ${ }^{1}$ Indian Institute of Mechanics of Continua, 201, Maniktala Main Road, \\ Suite No. 42, Calcutta 700 054, India \\ ${ }^{2}$ Department of Mathematics, University of Kalyani, Kalyani 741235 , India
}

MS received 3 April 1997; revised 30 April 1998

\begin{abstract}
The aim of this paper is to investigate magneto-thermo-viscoelastic surface waves in electrically and thermally conducting layers involving time rates of strain and stress of order $n$, the media being under an initial stress in the nature of hydrostatic tension or compression. The theory of magnetothermo-visco-elastic surface waves in the conducting medium involving strain rate and stress rate of $n$th order is derived under initial stress. This theory is then employed to obtain wave velocity equations in specific cases. Results obtained in the above cases reduce to well-known classical results when additional fields are absent.
\end{abstract}

Keywords. Magneto-thermo-visco-elasticity; visco-elasticity; surface waves; conducting layer; hydrostatic tension compression.

\section{Introduction}

In recent years, the interaction of Maxwell's electromagnetic fields with the deformation and propagation of waves in conducting solids has been receiving greater attention (for example, Knopoff 1955, Banos 1956, Chadwick 1957, Ewing et al 1957, Willson 1963, De \& Sengupta 1971, etc). Owing to its theoretical and practical importance in different branches of science, technology and engineering, Yu \& Tang (1966) thoroughly studied the dilatational and rotational waves in magneto-elastic initially stressed conductors. They considered three particular cases of initial stress, viz. (a) hydrostatic tension or compression, (b) uniaxial tension or compression and (c) uniform shear stress. De \& Sengupta (1971, 1972) investigated magneto-elastic waves and disturbances in initially stressed conducting media. Recently accommodation of thermal fields in the above kind of interaction is receiving greater attention from many investigators (Nowacki 1962; Paria 1962; Willson 1963; Bakshi 1970; Acharya \& Sengupta 1978). The effect of viscosity of first order of the conducting media with interaction of the magnetic field has been studied by Das et al 
(1994, pp 243-255). A limited discussion can also be found in the works of some applied scientists (Knopoff 1955; Flugge 1967; Roy \& Sengupta 1985; Das \& Sengupta 1990). However, in all the previous cases these effects have been studied either separately or in particular types of problems.

In the present paper, the authors have investigated and reviewed the joint effect of magnetic field, thermal field and initial stress in the form of hydrostatic tension or compression. The viscous nature of the media has been characterised by strain and stress. rates of order $n$, and the waves are propagated in such layers. It is assumed in the present case that heat is radiated from the free boundary surfaces of the layers, the maximum temperature difference across these surfaces always being small (Carslaw \& Jaeger 1959; Chadwick 1960). In particular, boundaries are assumed to be thermally insulated (Nowacki 1962).

The practical importance of the investigation presented here is that we may apply our results to the surface waves on Earth. The classical theories of propagation of waves in an elastic layer are very restricted and simplified. However, if the material of the Earth is supposed to be initially stressed and conducting, the above classical theories need proper modification. The inner parts of the Earth are under considerable stress from the weight of the matter resting on its surface. This causes a certain amount of deformation and so the Earth is initially stressed. We may suppose that the initial equilibrium stress is approximately of hydrostatic nature. Moreover, the Earth has its own magnetic field and a thermal field also inside it. Besides these, the material medium of the Earth may be considered visco-elastic in general, in some places satisfying strain and stress rates of order $n$.

Variations of the wave velocity in a layer due to the above fields are sometimes not of practical importance if the concerned fields are sufficiently weak. However, if the concerned fields and the corresponding effects are significant then the variations must be taken into account. These criteria are considered in the discussion part of the problem in the present paper. It is believed that the present problem has not been investigated earlier.

\section{Basic equations}

In the absence of displacement current, Maxwell's equations for homogeneous isotropic material (strictly speaking, the material will not remain homogeneous and isotropic when it is subjected to initial stress, magnetic field and thermal field; we, however, ignore such variations in the present investigation) may be written as follows:

$$
\begin{aligned}
& \nabla \times \mathbf{H}=\mathbf{J}, \nabla \times \mathbf{E}=-\partial \mathbf{B} / \partial t \\
& \nabla \cdot \mathbf{B}=0, \mathbf{B}=\mu_{e} \mathbf{H}
\end{aligned}
$$

and Ohm's law (Bakshi 1970) is

$$
\mathbf{J}=\sigma[\mathbf{E}+(\partial \mathbf{u} / \partial t) \times \mathbf{B}]-k_{0} \nabla T,
$$

where $\mathbf{E}$ and $\mathbf{H}$ are the electric and the magnetic intensity vectors. $\mathbf{B}$ denotes the magnetic induction vector, $\mathbf{J}$ the current density vector, $\mu_{e}$ the magnetic permeability, $\sigma$ the electric conductivity, $k_{0}$ the linking electric field with temperature gradient, $\mathbf{u}$ the displacement vector, $T$ the absolute temperature over initial absolute temperature $T_{0}$ and $t$ denotes time. 
The $n$th order stress strain relation for an isotropic visco-elastic medium under temperature field when both strain and stress rates are taken into account is (Flugge 1967; Nowacki 1962; Pal \& Sengupta 1987)

$$
D_{\eta} \tilde{\tau}_{j k}=2 D_{\mu} e_{j k}+\left[D_{\lambda} e-D_{\beta} T\right] \delta_{j k},
$$

where

$$
\begin{aligned}
& D_{\eta}=\eta_{1}+\eta_{2} \frac{\partial}{\partial t}+\eta_{3} \frac{\partial^{2}}{\partial t^{2}}+\cdots+\eta_{n+1} \frac{\partial^{n}}{\partial t^{n}} \\
& D_{\mu}=\mu_{1}+\mu_{2} \frac{\partial}{\partial t}+\mu_{3} \frac{\partial^{2}}{\partial t^{2}}+\cdots+\mu_{n+1} \frac{\partial^{n}}{\partial t^{n}} \\
& D_{\lambda}=\lambda_{1}+\lambda_{2} \frac{\partial}{\partial t}+\lambda_{3} \frac{\partial^{2}}{\partial t^{2}}+\cdots+\lambda_{n+1} \frac{\partial^{n}}{\partial t^{n}} \\
& D_{\beta}=\beta_{1}+\beta_{2} \frac{\partial}{\partial t}+\beta_{3} \frac{\partial^{2}}{\partial t^{2}}+\cdots+\beta_{n+1} \frac{\partial^{n}}{\partial t^{n}}
\end{aligned}
$$

and, $\eta_{1}, \lambda_{1}, \mu_{1}, \beta_{1}$ stand for classical physical parameters whereas $\eta_{i}, \lambda_{i}, \mu_{i}, \beta_{i}$ $[i=2,3, \ldots, n+1]$ denote the physical constants of order $n$. Also,

$$
\begin{aligned}
e_{j k} & =\frac{1}{2}\left(u_{j, k}+u_{k, j}\right), \\
\beta_{i} & =\alpha_{t}\left(3 \lambda_{i}+2 \mu_{i}\right)
\end{aligned}
$$

$\alpha_{t}$ being the coefficient of linear thermal expansion.

The generalised Fourier Law of heat conduction (in the absence of a heat source) is given by (Paria 1962)

$$
k \nabla^{2} T=\rho c_{v} \frac{\partial T}{\partial t}+T_{0} D_{\beta} \frac{\partial e}{\partial t}+\pi_{0} \nabla \cdot \mathbf{J},
$$

where $k$ is the coefficient of heat conduction, $\rho$ denotes density of the material, $C_{v}$ is the specific heat of the body at constant volume, $\pi_{0}$ is the coefficient linking the current velocity with that of heat flow and $e$ is dilatation.

For electrically as well as thermally conducting charge-free thermoelastic solid under an initial stress in an electro-magnetic field, the equation of motion, based on the finite strain theory when the body is subjected to small perturbation is (Yu Tang 1966; Bakshi 1970)

$$
\rho \frac{\partial^{2} u_{i}}{\partial t^{2}}=\frac{\partial}{\partial x_{j}}\left[\tau_{j k}\left(\delta_{j k}+\frac{\partial u_{i}}{\partial x_{k}}\right)\right]+(\mathbf{J} \times \mathbf{B})_{i}+F_{i}
$$

where

$$
\begin{aligned}
\tau_{j k} & =\tau_{j k}\left(x_{1}, x_{2}, x_{3}\right)+\tilde{\tau}_{j k}\left(x_{1}, x_{2}, x_{3}, t\right), \\
F_{i} & =F_{i}^{0}\left(x_{1}, x_{2}, x_{3}\right)+\tilde{F}_{i}\left(x_{1}, x_{2}, x_{3}, t\right), \\
H_{i} & =H_{i}\left(x_{1}, x_{2}, x_{3}\right)+\tilde{H}_{i}\left(x_{1}, x_{2}, x_{3}, t\right), i, j, k=1,2,3,
\end{aligned}
$$

in which the superscript ' 0 ' corresponds to the initial equilibrium state and $\tilde{\tau}_{j k}, \tilde{F}_{i}, \tilde{H}_{i}$ are the corresponding perturbed quantities with respect to the corrdinates $\left(x_{1}, x_{2}, x_{3}\right)$ and time $t$. 
From (1) and (2) we have,

$$
\frac{\partial \mathbf{H}}{\partial t}=\frac{1}{\mu_{2}} \nabla \times\left[\mu_{e} \frac{\partial \mathbf{u}}{\partial t} \times \mathbf{H}-\frac{1}{\sigma} \nabla \times \mathbf{H}\right] .
$$

Again (6) may be written as

$$
\rho \frac{\partial^{2} u_{i}}{\partial t^{2}}=\frac{\partial}{\partial x_{j}}\left[\tau_{j k}\left(\delta_{j k}+\frac{\partial u_{i}}{\partial x_{k}}\right)\right]+\mu_{e}[(\nabla \times \mathbf{H}) \times \mathbf{H}]+\mathbf{F}_{i}
$$

Equations (7) \& (8) are formally identical to the corresponding equations obtained by Yu \& Tang (1966) for the magneto-elastic case. Hence following the same process of approximation as Yu \& Tang (1966) and Acharya \& Sengupta (1978) we arrive at the same set of equations of motion for a perfectly conducting thermo-elastic solid under the hydrostatic tension or compression in a uniform magnetic field as follows:

$$
\begin{aligned}
\rho \frac{\partial^{2} u_{i}}{\partial t^{2}} & =-p_{0} \frac{\partial^{2} u_{i}}{\partial x_{j} \partial x_{j}}+\mu_{e} H_{0}\left(\frac{\partial \tilde{H}_{i}}{\partial x_{1}}-\frac{\partial \tilde{H}_{1}}{\partial x_{i}}\right)+\frac{\partial \tilde{\tau}_{i j}}{\partial x_{j}}, \\
\tilde{H}_{i} & =H_{0}\left(\frac{\partial u_{i}}{\partial x_{1}}-\frac{\partial u_{1}}{\partial x_{i}}\right),
\end{aligned}
$$

where $-p_{0}$ is hydrostatic tension or compression according as $p_{0}<0$ or $p_{0}>0$ and $\tilde{\tau}_{i j}$ is given by (3) and $H_{0}$ is the intensity of the uniform magnetic field parallel to the $x_{1}$ axis.

\section{Formulation of the problem}

Let us consider a homogeneous, isotropic, visco-elastic, conducting (both electrically and thermally) solid layer including strain and stress rates of finite thickness $2 H$ under an initial hydrostatic tension or compression permeated by uniform magnetic and thermal fields. Let us introduce an orthogonal Cartesian coordinate system $O x_{1} x_{2} x_{3}$, the origin $O$ being any point on the middle plane of the infinite plate and $O x_{3}$ being a line drawn vertically downwards. There exists a plane wave moving with a constant velocity $C$ in the $x_{1}$ direction. From the nature of the problem we assume that all causes and effects depend on two space variables $x_{1}$ and $x_{3}$ and time $t$. So we assume displacement vector $\mathbf{u}$ in the form

$$
\mathbf{u}=\left(u_{1}, O, u_{3}\right), \quad u_{i}=u_{i}\left(x_{1}, x_{3}, t\right), \quad i=1,3 .
$$

We now introduce the displacement potentials $\Phi\left(x_{1}, x_{3}, t\right)$ and $\Psi\left(x_{1}, x_{3}, t\right)$ where,

$$
\begin{aligned}
& u_{1}=\frac{\partial \Phi}{\partial x_{1}}-\frac{\partial \Psi}{\partial x_{3}} \\
& u_{3}=\frac{\partial \Phi}{\partial x_{3}}+\frac{\partial \Psi}{\partial x_{1}}
\end{aligned}
$$

Hence we obtain,

$$
\begin{aligned}
& \nabla^{2} \Phi=e, \quad \nabla^{2} \Psi=\frac{\partial u_{3}}{\partial x_{1}}-\frac{\partial u_{1}}{\partial x_{3}} \\
& \nabla^{2} \equiv \frac{\partial^{2}}{\partial x_{1}^{2}}+\frac{\partial^{2}}{\partial x_{3}^{2}}, \quad e=\frac{\partial u_{1}}{\partial x_{1}}+\frac{\partial u_{3}}{\partial x_{3}}
\end{aligned}
$$


Now introducing (3) into (9) we obtain the displacement equations of motion for perfectly conducting visco-elastic medium of $n$th order under hydrostatic tension or compression in an uniform magnetic as well as thermal field as follows:

$$
\begin{gathered}
\rho D_{\eta} \frac{\partial^{2} u_{1}}{\partial t^{2}}=\left(D_{i}+D_{\mu}\right) \frac{\partial e^{3}}{\partial x_{1}}+D_{\mu} \nabla^{2} u_{1}-p_{0} D_{\eta} \nabla^{2} u_{1}-D_{\beta} \frac{\partial T}{\partial x_{1}} \\
\rho D_{\eta} \frac{\partial^{2} u_{3}}{\partial t^{2}}=\left(D_{\lambda}+D_{\mu}\right) \frac{\partial e^{2}}{\partial x_{3}}+D_{\mu} \nabla^{2} u_{3}-p_{0} D_{\eta} \nabla^{2} u_{3} \\
+K D_{\eta}\left(\frac{\partial^{2} u_{3}}{\partial x_{1}^{2}}-\frac{\partial^{2} u_{1}}{\partial x_{1} \partial x_{3}}\right)-D_{\beta} \frac{\partial T}{\partial x_{3}}
\end{gathered}
$$

where

$$
k=\mu_{c} H_{0}^{2}
$$

Now introducing (11) and (12) into (13) we get

$$
\begin{aligned}
& \frac{\partial^{2} \Phi}{\partial t^{2}}=\left(M_{L}-\frac{p_{0}}{\rho}\right) \nabla^{2} \Phi+\frac{K}{\rho} \cdot \frac{\partial^{2} \Psi}{\partial x_{1} \partial x_{3}}-\frac{M_{T}}{\rho} \cdot T . \\
& \frac{\partial^{2} \Psi}{\partial t^{2}}=\left(M_{S}-\frac{p_{0}}{\rho}\right) \nabla^{2} \Psi+\frac{K}{\rho} \cdot \frac{\partial^{2} \Psi}{\partial x_{1}^{2}} .
\end{aligned}
$$

in which.

$$
\begin{aligned}
& M_{L}=\left(D_{\lambda}+2 D_{\mu}\right) /\left(\rho D_{\eta}\right) \\
& M_{S}=D_{\mu} /\left(\rho D_{\eta}\right) \\
& M_{T}=D_{\beta} / D_{\eta} .
\end{aligned}
$$

Equation (5) in view of (1), (11) and (12) may be written as,

$$
\nabla^{2} T=\frac{\rho C_{l}}{k}-\frac{\partial T}{\partial t}+\frac{T_{0}}{k} \cdot D_{\beta} \frac{\partial}{\partial t}\left(\nabla^{2} \Phi\right) .
$$

\subsection{Boundary conditions}

For the present problem the boundary surfaces $\left(x_{3}= \pm H\right)$ of the layer are free from stress, i.e.,

$$
\tilde{\tau}_{13}=0=\tilde{\tau}_{3,3}, \quad \text { at } \quad x_{3}= \pm H
$$

By (11) \& (12) we obtain the stress components from (3) as follows:

$$
\begin{aligned}
& \tilde{\tau}_{13}=\rho M_{S}\left[2 \frac{\partial^{2} \Phi}{\partial x_{1} \partial x_{3}}-\frac{\partial^{2} \Psi}{\partial x_{3}^{2}}+\frac{\partial^{2} \Psi}{\partial x_{1}^{2}}\right] . \\
& \tilde{\tau}_{33}=\rho M_{L} \nabla^{2} \Phi-2 \rho M_{S}\left[\frac{\partial^{2} \Phi}{\partial x_{1}^{2}}-\frac{\partial^{2} \Psi}{\partial x_{1} \partial x_{3}}\right]-M_{T} T .
\end{aligned}
$$


Since the temperature difference across the free surface is always small, the linearised form of radiative condition is valid (Carslaw \& Jaeger 1959; Chadwick 1960) on each of the boundaries $x_{3}= \pm H$.

Clearly, at $x_{3}= \pm H$ we have

$$
\frac{\partial T}{\partial x_{3}}+h T=0,
$$

where $h$ is Planck's constant.

\section{Solution of the problem}

For simple harmonic waves and corresponding temperature fluctuations, we seek the solutions of (14) and (16) in the following form:

$$
\begin{aligned}
& \Phi\left(x_{1}, x_{3}, t\right)=\Phi^{*}\left(x_{3}\right) \cdot \exp \left[i\left(\omega t-\eta x_{1}\right)\right] \\
& \Psi\left(x_{1}, x_{3}, t\right)=\Psi^{*}\left(x_{3}\right) \cdot \exp \left[i\left(\omega t-\eta x_{1}\right)\right] \\
& T\left(x_{1}, x_{3}, t\right)=T^{*}\left(x_{3}\right) \cdot \exp \left[i\left(\omega t-\eta x_{1}\right)\right]
\end{aligned}
$$

Introducing (20) in (14) and (16) we obtain,

$$
\begin{aligned}
& {\left[\frac{\mathrm{d}^{2}}{\mathrm{~d} x_{3}^{2}}-\eta^{2}+\frac{\omega^{2}}{M_{L}^{*}}\right] \Phi^{*}=\frac{i \eta k}{\rho M_{L}^{*}} \cdot \frac{\mathrm{d} \Psi^{*}}{\mathrm{~d} x_{3}}+\frac{M_{T}^{*}}{\rho M_{L}^{*}} T^{*},} \\
& {\left[\frac{\mathrm{d}^{2}}{\mathrm{~d} x_{3}^{2}}-\eta^{2}+\frac{\omega^{2}}{M_{S}^{*}}\right] \Psi^{*}=\frac{K \eta^{2}}{\rho M_{S}^{*}} \Psi^{*},} \\
& {\left[\frac{\mathrm{d}^{2}}{\mathrm{~d} x_{3}^{2}}-\eta^{2}-\frac{\rho C_{v} i \omega}{k}\right] T^{*}=\frac{i \omega T_{0} D_{\beta}^{*}}{k}\left(\frac{\mathrm{d}^{2}}{\mathrm{~d} x_{3}^{2}}-\eta^{2}\right) \Phi^{*},}
\end{aligned}
$$

where

$$
\begin{aligned}
& M_{L}^{*}=\frac{D_{\lambda}^{*}+2 D_{\mu}^{*}}{\rho D_{\eta}^{*}}-\frac{p_{0}}{\rho}, \\
& M_{S}^{*}=\frac{D_{\mu}^{*}}{\rho D_{\eta}^{*}}-\frac{p_{0}}{\rho}, \quad M_{T}^{*}=\frac{D_{\beta}^{*}}{D_{\eta}^{*}},
\end{aligned}
$$

and

$$
\begin{array}{ll}
D_{\eta}^{*}=\sum_{j=0}^{n} \eta_{j+1}(i \omega)^{j}, & D_{\lambda}^{*}=\sum_{j=0}^{n} \lambda_{j+1}(i \omega)^{j} \\
D_{\mu}^{*}=\sum_{j=0}^{n} \mu_{j+1}(i \omega)^{j}, & D_{\beta}^{*}=\sum_{j=0}^{\eta} \beta_{j+1}(i \omega)^{j} .
\end{array}
$$


Clearly, solutions of (21) must have exponential order. The solutions of (21) may be taken as,

$$
\begin{aligned}
\Phi^{*}= & A \sinh \zeta_{1} x_{3}+B \cosh \zeta_{1} x_{3}+c \sinh \zeta_{2} x_{3}+D \cosh \zeta_{2} x_{3} \\
& +E \sinh \zeta_{3} x_{3}+F \cosh \zeta_{3} x_{3}, \\
\Psi^{*}= & E_{1} \sinh \zeta_{3} x_{3}+F_{1} \cosh \zeta_{3} x_{3}, \\
T^{*}=A_{2} & \sinh \zeta_{1} x_{3}+B_{2} \cosh \zeta_{1} x_{3}+C_{2} \sinh \zeta_{2} x_{3}+D_{2} \cosh \zeta_{2} x_{3} \\
& +E_{2} \sinh \zeta_{3} x_{3}+F_{2} \cosh \zeta_{3} x_{3},
\end{aligned}
$$

where $\zeta_{i}^{2}=\eta^{2}-\delta_{i}^{2}, i=1,2,3$. In which $\delta_{1}^{2}, \delta_{2}^{2}$ are the solutions of

$$
\delta^{4}-\delta^{2}\left[\frac{\omega^{2}}{M_{L}^{*}}-\frac{i \omega \rho C_{v}}{k}(1+\varepsilon)\right]-\frac{i \omega^{3} \rho C_{v}}{k M_{L}^{*}}=0
$$

and

$$
\delta_{3}^{2}=\frac{\omega^{2}}{M_{S}^{*}}-\frac{K \eta^{2}}{\rho M_{S}^{*}}, \quad \varepsilon=\frac{T_{0} M_{T}^{*} D_{\beta}^{*}}{\rho^{2} C_{v} M_{L}^{*}},
$$

and the constants in the solutions (23) are related by,

$$
\begin{aligned}
& A_{2}=\gamma_{1} A, \quad B_{2}=\gamma_{1} B, \quad C_{2}=\gamma_{2} C, \quad D_{2}=\gamma_{2} D, \\
& E_{2}=\gamma_{3} E, \quad F_{2}=\gamma_{3} F, \quad E=m F_{1}, \quad F=m E_{1} \text {, }
\end{aligned}
$$

where,

$$
\begin{aligned}
\gamma_{j}= & =\frac{\rho M_{L}^{*}}{M_{T}^{*}}\left(\frac{\omega^{2}}{M_{L}^{*}}-\delta_{j}^{2}\right), \quad j=1,2, \\
\gamma_{3}= & \left(i \omega T_{0} D_{\beta}^{*} \delta_{3}^{2}\right) /\left[k\left(\frac{\rho C_{v} i \omega}{k}+\delta_{3}^{2}\right)\right], \\
m= & {\left[\left(\frac{\rho C_{v} i \omega}{k}+\delta_{3}^{2}\right) \frac{i \eta k}{M_{T}^{*}}\left(\eta^{2}-\delta_{3}^{2}\right)^{1 / 2}\right] \div } \\
& {\left[\frac{\rho M_{L}^{*}}{M_{T}^{*}}\left(\frac{\rho C_{v} i \omega}{k}+\delta_{3}^{2}\right)\left(\frac{\omega^{L}}{M_{L}^{*}}-\delta_{3}^{2}\right)-\frac{i \omega T_{0} D_{\beta}^{*} \delta_{3}^{2}}{k}\right] . }
\end{aligned}
$$

In evaluating quantities like $\left(\eta^{2}-\delta_{3}^{2}\right)^{1 / 2}$ the root with positive real part must be taken in each case.

Hence, (20) and (23) together give the solution of $\Phi, \Psi, T$.

Now employing the boundary conditions (17) and (19) to (20) and (23) we get

$$
\begin{aligned}
& A \xi_{1} p_{1}-B \alpha_{1} p_{1}+c \xi_{2} p_{2}-D \alpha_{2} p_{2}-E_{1} \xi_{3} p_{3}+F_{1} \alpha_{3} p_{3}=0, \\
& A \alpha_{1} q_{1}-B \xi_{1} q_{1}+c \alpha_{2} q_{2}-D \xi_{2} q_{2}-E_{1} \alpha_{3} q_{3}+F_{1} \xi_{3} q_{3}=0, \\
& A n_{1} p_{1}-B v_{1} p_{1}+c n_{2} p_{2}-D \nu_{2} p_{2}-E_{1} n_{3} p_{3}+F_{1} \nu_{3} p_{3}=0, \\
& A \nu_{1} q_{1}-B n_{1} q_{1}+c v_{2} q_{2}-D n_{2} q_{2}-E_{1} \nu_{3} q_{3}+F_{1} n_{3} q_{3}=0, \\
& A s_{1} p_{1}-B r_{1} p_{1}+c s_{2} p_{2}-D r_{2} p_{2}-E_{1} s_{3} p_{3}+F_{1} r_{3} p_{3}=0, \\
& A r_{1} q_{1}-B s_{1} q_{1}+c r_{2} q_{2}-D s_{2} q_{2}-E_{1} r_{3} q_{3}+F_{1} s_{3} q_{3}=0,
\end{aligned}
$$


where,

$$
p_{j}=\sinh \zeta_{j} H, \quad q_{j}=\cosh \xi_{j} H, \quad j=1,2,3
$$

and

$$
\begin{aligned}
& \xi_{1}= 0=\xi_{2}, \quad \xi_{3}=\eta^{2}+\zeta_{3}^{2}+z i m \eta \xi_{3}, \\
& \alpha_{j}= z i \eta \xi_{j}(j=1,2), \quad \alpha_{3}=0, \\
& \nu_{1}= 0=\nu_{2}, \quad n_{3}=0, \\
& \nu_{3}= m \rho\left(M_{L}^{*}+\frac{p_{0}}{\rho}\right)\left(\eta^{2}-\zeta_{3}^{2}\right) \\
& \quad-2 \rho\left(M_{S}^{*}+\frac{p_{0}}{\rho}\right)\left(m \eta^{2}-i \eta \zeta_{3}\right)+m \zeta_{3} M_{T}^{*}, \\
& n_{j}=\rho\left(M_{L}^{*}+\frac{p_{0}}{\rho}\right)\left(\eta^{2}-\zeta_{j}^{2}\right)+2 \rho\left(M_{S}^{*}+\frac{p_{0}}{\rho}\right) \eta^{2}+\gamma_{j} M_{T}^{*}, \quad(j=1,2), \\
& s_{j}=-h \gamma_{j}, \quad r_{j}=\gamma_{j} \zeta_{j}, \quad(j=1,2), \\
& s_{3}=m \zeta_{3} \gamma_{3}, \quad r_{3}=-m h \gamma_{3} .
\end{aligned}
$$

Now eliminating $A, B, C, D, E_{1}, F_{1}$ from (26) we have the following determinantal equation:

$$
\Delta=\left|\begin{array}{cccccc}
\xi_{1} & \alpha_{1} & \xi_{2} \frac{y_{2}}{y_{1}} & \alpha_{2} \frac{y_{2}}{y_{1}} & \xi_{3} \frac{y_{3}}{y_{1}} & \alpha_{3} \frac{y_{3}}{y_{1}} \\
n_{1} \frac{y_{1}}{y_{2}} & v_{1} \frac{y_{1}}{y_{2}} & n_{2} & v_{2} & n_{3} \frac{y_{3}}{y_{2}} & v_{3} \frac{y_{3}}{y_{2}} \\
s_{1} \frac{y_{1}}{y_{3}} & r_{1} \frac{y_{1}}{y_{3}} & s_{2} \frac{y_{2}}{y_{3}} & r_{2} \frac{y_{2}}{y_{3}} & s_{3} & r_{3} \\
\alpha_{1} & \xi_{1} & \alpha_{2} & \xi_{2} & \alpha_{3} & \xi_{3} \\
v_{1} & n_{1} & v_{2} & n_{2} & v_{3} & n_{3} \\
r_{1} & s_{1} & r_{2} & s_{2} & r_{3} & s_{3}
\end{array}\right|
$$

where,

$$
y_{j}=\tanh \zeta_{j} H, \quad j=1,2,3 \text {. }
$$

\section{Particular cases}

Case 1: Let us consider the particular case in which symmetry and asymmetry of stresses and displacement components are taken into consideration and for which we take,

$$
\begin{aligned}
\Phi_{1}= & \left(B \cosh \zeta_{1} x_{3}+D \cosh \zeta_{2} x_{3}+m E_{1} \cosh \zeta_{3} x_{3}\right) \times \exp \left[i\left(\omega t-\eta x_{1}\right)\right] \\
\Psi_{1}= & \left(E_{1} \sinh \zeta_{3} x_{3}\right) \times \exp \left[i\left(\omega t-\eta x_{1}\right)\right], \\
T_{1}= & \left(\gamma_{1} B \cosh \zeta_{1} x_{3}+\gamma_{2} D \cosh \zeta_{2} x_{3}+m \gamma_{3} E_{1} \cosh \zeta_{3} x_{3}\right) \\
& \quad \times \exp \left[i\left(\omega t-\eta x_{1}\right)\right] .
\end{aligned}
$$


Introducing (31) in (11) and (18) we see that the displacements $u_{1}$ and $u_{3}$ and the stress component $\tilde{\tau}_{33}$ are symmetric with respect to the plane $x_{3}=0$, while the stress component $\tilde{\tau}_{13}$ is antisymmetric with respect to the same plane. On the boundary, we have

$$
\begin{aligned}
& \tilde{\tau}_{13}=0, \\
& \tilde{\tau}_{33}=0,
\end{aligned}
$$

in the plane $x_{3}= \pm H$.

Further, we assume that boundaries are thermally insulated, so that in this particular case we have $h=0$ (Nowacki 1962) and hence

$$
\partial T_{1} / \partial x_{3}=0, \quad \text { on } \quad x_{3}= \pm H
$$

Applying boundary conditions (32) and (33) into (31) we get,

$$
\begin{aligned}
& B \alpha_{1} p_{1}+D \alpha_{2} p_{2}+E_{1} \xi_{3} p_{3}=0, \\
& B n_{1} q_{1}+D n_{2} q_{2}+E_{1} \nu_{3} q_{3}=0, \\
& B r_{1} p_{1}+D r_{2} p_{2}+E_{1} s_{3} p_{3}=0,
\end{aligned}
$$

where all the notations are defined in (25), (27), (28).

Eliminating $B, D, E_{1}$ from (34) we get the following equation after a little simplification,

$$
\frac{\tanh \zeta_{1} H}{\tanh \zeta_{2} H}=-L /\left[P+Q \frac{\tanh \zeta_{2} H}{\tanh \zeta_{3} H}\right]
$$

where,

$$
\begin{aligned}
& L=n_{1}\left(r_{2} \xi_{3}-\alpha_{2} s_{3}\right), \\
& P=n_{2}\left(\alpha_{1} s_{3}-r_{1} \xi_{3}\right), \\
& Q=v_{3}\left(r_{1} \alpha_{2}-r_{2} \alpha_{1}\right) .
\end{aligned}
$$

Case 2: We now consider a special case of displacement potential and temperature field as explained in the following section and for which we take

$$
\begin{aligned}
\Phi_{2}= & \left(A \sinh \zeta_{1} x_{3}+C \sinh \zeta_{2} x_{3}+m F_{1} \sinh \zeta_{3} x_{3}\right) \times \exp \left[i\left(\omega t-\eta x_{1}\right)\right] \\
\Psi_{2}= & \left(F_{1} \cosh \zeta_{3} x_{3}\right) \times \exp \left[i\left(\omega t-\eta x_{1}\right)\right] \\
T_{2}= & \left(\gamma_{1} A \sinh \zeta_{1} x_{3}+\gamma_{2} C \sinh \zeta_{2} x_{3}+m \gamma_{3} F_{1} \sinh \zeta_{3} x_{3}\right) \\
& \quad \times \exp \left[i\left(\omega t-\eta x_{1}\right)\right] .
\end{aligned}
$$

Introducing (37) in (11) and (18) we see that the displacement components $u_{1}$ and $u_{3}$ and the stress components $\tilde{\tau}_{33}$ are anti-symmetric with respect to the plane $x_{3}=0$, whereas $\tilde{\tau}_{13}$ is symmetric with respect to the same plane.

Proceeding in the same way as in case 1 , we obtain the following wave-velocity transcedental equation.

$$
\frac{\tanh \zeta_{1} H}{\tanh \zeta_{2} H}=-\left[P+Q \cdot \frac{\tanh \zeta_{3} H}{\tanh \zeta_{2} H}\right] / L,
$$

where $L, P, O$ are the same as in (36). 


\section{Various situations and discussions}

The determinantal equation (29) represents the wave velocity equation of the waves propagated in a magneto-thermo-visco-elastic conducting layer including strain and stress rates of order $n$ permeated by a uniform magnetic field parallel to the $x_{1}$-axis and a uniform thermal field as well as under the hydrostatic tension or compression. It is easy to see that this wave-propagation essentially depends on the magnetic and thermal fields and also on the initial stress and visco-elastic nature of the material medium.

First, assuming a situation where the wavelength is very small in comparison with the thickness $2 H$ of the layer, the quantities $\zeta_{j} H(j=1,2,3)$ and $\eta H$ become so large that the ratios of the hyperbolic tangents in (29) are approximately unity and hence (29) reduces to

$$
\Delta_{1} \cdot \Delta_{2}=0
$$

i.e. either

$$
\Delta_{1}=0 \text { or } \Delta_{2}=0
$$

where

$$
\Delta_{1}=\left|\begin{array}{ccc}
\xi_{1}+\alpha_{1} & \xi_{2}+\alpha_{2} & \xi_{3}+\alpha_{3} \\
n_{1}+v_{1} & n_{2}+\nu_{2} & n_{3}+\nu_{3} \\
s_{1}+\gamma_{1} & s_{2}+\gamma_{2} & s_{3}+\gamma_{3}
\end{array}\right|,
$$

and

$$
\Delta_{2}=\left|\begin{array}{ccc}
\xi_{1}-\alpha_{1} & \xi_{2}-\alpha_{2} & \xi_{3}-\alpha_{3} \\
n_{1}-v_{1} & n_{2}-v_{2} & n_{3}-\nu_{3} \\
s_{1}-\gamma_{1} & s_{2}-\gamma_{2} & s_{3}-\gamma_{3}
\end{array}\right|
$$

Here $\Delta_{1}=0$ represents the wave-velocity equation for the magneto-thermo-viscoelastic Rayleigh waves under the initial stress of hydrostatic tension or compression involving both strain rate and stress rate of order $n$, propagating in a semi-infinite medium having a plane horizontal boundary in the uppermost part of the solid. On the other hand, if the semi-infinite body has a plane boundary at its lowest part, then $\Delta_{2}=0$ is the corresponding wave-velocity equation. Although the thickness of the layer is assumed to be finite and large, in the case of Rayleigh waves it has to be considered that it is a semiinfinite medium having a plane boundary at the uppermost or at the lowest part and for this, the Rayleigh wave-velocity equation occurs twice, depending on the whether the free plane boundary is at the top or the bottom of the medium respectively. In the first case, the medium is extended to infinity at the lower part where $\Delta_{1}=0$ represents the type of wave propagating in the vicinity of the free upper plane boundary. The latter is just the reverse of this, where $\Delta_{2}=0$ signifies the nature of Rayleigh waves propagating in the vicinity of the free lower plane boundary when the medium is extended towards infinity at the top. It is evident from the mathematical form of the two equations $\Delta_{1}=0, \Delta_{2}=0$ that they are inter-changable simply by converting $\zeta_{j}$ to $-\zeta_{j}(j=1,2,3)$, i.e. by changing the direction of the $x_{3}$-axis. This explains the existence of two wave-velocity equations. 
Let us first study the equation $\Delta_{1}=0 ; \Delta_{2}=0$ may then be treated in similar fashion. As the equation $\Delta_{1}=0$ contains $\zeta$, the magneto-thermo-visco-elastic Rayleigh waves in initially stressed medium is subjected to dispersion of general waveform.

In the absence of initial stress and magnetic field $\left(p_{0}=0, k=0\right)$, the determinantal equation $\Delta_{1}=0$ reduces to

$$
\begin{gathered}
\left(2-\frac{c^{2}}{M_{S}^{*}}\right)^{2}\left(\sigma_{1}^{2}+\sigma_{1} \sigma_{2}+\sigma_{2}^{2}-1+\frac{c^{L}}{M_{L}^{*}}\right)-4 \sigma_{1} \sigma_{2} \sigma_{3}\left(\sigma_{1}+\sigma_{2}\right) \\
=\frac{h}{\eta}\left[\left(2-\frac{c^{L}}{M_{S}^{*}}\right)^{2}\left(\sigma_{1}+\sigma_{2}\right)-4 \sigma_{3}\left(\sigma_{1} \sigma_{2}+1-\frac{c^{2}}{M_{L}^{*}}\right)\right],
\end{gathered}
$$

where $\sigma_{j}^{2}=1-\delta_{j}^{2} / \eta^{2}, j=1,2,3, c=$ velocity of wave $=\omega / \eta$, and

$$
M_{L}^{*}=\frac{D_{\lambda}^{*}+2 D_{\mu}^{*}}{\rho D_{\eta}^{*}}, M_{S}^{*}=\frac{D_{\mu}^{*}}{\rho D_{\eta}^{*}} .
$$

The equation (41) is the wave-velocity equation for the $n$th order thermo-visco-elastic Rayleigh waves (Das \& Sengupta 1990). Moreover, if we neglect the effect of viscosity we get a result similar to (41), the only difference being,

$$
M_{L}^{*}=\frac{\lambda_{1+2} \mu}{\rho}, M_{S}^{*}=\frac{\mu_{1}}{\rho},
$$

and this result is in agreement with the corresponding result obtained by Chadwick (1960) under thermo-elastic conditions.

If we neglect the effect of temperature and consider stress and strain rates of order $n$ then we have from (40),

$$
\Delta_{1}=\left|\begin{array}{ll}
\xi_{1}+\alpha_{1} & \xi_{3}+\alpha_{3} \\
n_{1}+l_{1} & n_{3}+\nu_{3}
\end{array}\right|=0
$$

On simplification this yields,

$$
\begin{aligned}
\left(2-\frac{c^{L}}{M_{S}^{*}}\right)^{2}+\left(2-\frac{c^{L}}{M_{S}^{*}}\right) \frac{K}{\rho M_{S}^{*}}-\frac{4 \zeta_{1} \zeta_{3}}{\eta^{2}} \\
+\frac{2 i \gamma_{1}}{\eta}\left(\zeta_{1}-\zeta_{3}\right)\left[\left(\frac{\zeta_{1} \zeta_{3}}{\eta^{2}}+1\right) \frac{M_{L}^{*}}{M_{S}^{*}}-2\right]=0 .
\end{aligned}
$$

This wave-velocity equation (42) corresponds to the $n$th order magneto-visco-elastic Rayleigh waves. We note that the equivalent result with $n=1$ is in perfect agreement with the result obtained by Das et al (1994).

In addition to the assumption made in (42), if we omit the viscosity effect and the strain and stress rates we get,

$$
\alpha_{1} v_{3}-n, \xi_{3}=0,
$$

which is the magneto-elastic Rayleigh wave velocity equation under initial stress of hydrostatic tension or compression and is in good agreement with the result obtained by Acharya \& Sengupta (1978). 
In (41), when there are no viscosity or thermal effects, we expect the Rayleigh wavevelocity equation to hold for elastic media. In this case, our result obtained from (41) is of the form

$$
\left(2-\frac{C^{L}}{M_{S}^{*}}\right)^{2}=4\left[\left(1-\frac{C^{L}}{M_{L}^{*}}\right)\left(1-\frac{C^{L}}{M_{S}^{*}}\right)\right]^{1 / 2},
$$

where $c=\omega / \eta, M_{L}^{*}=\left(\lambda_{1}+2 \mu_{1}\right) / \rho, M_{S}^{*}=\mu_{1} / \rho$ and agrees with the classical result of Rayleigh (1885).

Next, we consider the case for large wavelengths relative to the thickness of the layer. Here the arguments of the hyperbolic functions in (29) becomes small and we make the assumption that

$$
\tanh \zeta_{j} H \simeq \zeta_{j} H, \quad j=1,2,3 .
$$

Hence, the corresponding wave-velocity equation may be obtained from (29) by taking $y_{j}=\zeta_{j} H, j=1,2,3$.

For the particular case 1 , if the length of the wave is large when compared with the thickness of the layer by the above approximation, we have from (35)

$$
\frac{\zeta_{1}}{\zeta_{2}}=-L\left[P+Q \frac{\zeta_{2}}{\zeta_{3}}\right]
$$

If the layer is initially stress-free, the magnetic field as well as the thermal field are absent from (45) (setting $k=0 p_{0}=0, \gamma_{1}, \gamma_{3} \rightarrow 0, \sigma_{2} \rightarrow \alpha$ ) we get,

$$
C^{L} M_{L}^{*}=4 M_{S}^{*}\left(M_{L}^{*}-M_{S}^{*}\right) .
$$

Equation (46) is the classical equation determining the wave-velocity in a visco-elastic layer including both strain and stress rates of order $n$. If we neglect the viscosity effect, (46) determines the wave-velocity in the classical isotropic elastic layer (Lamb 1916; Bullen 1963).

Again, if length of the wave is very small compared to the thickness of the layer, the quantities $\zeta_{j} H(j=1,2,3), \eta H$ are large and the ratios of hyperbolic tangents in (35) approach infinity. Hence from (35) we get,

$$
P+Q+L=0
$$

which is the equation determining the wave-velocity of Rayleigh surface waves in a magneto-thermo-visco-clastic initially stressed conducting (thermally and electrically) layer whose boundary is thermally insulated and which involves the stress and strain rates of order $n$. This result is in agreement with the result $\Delta_{1}=0$ in (39) obtained for $h=0$.

When $p_{o}=0, k=0$, i.e. the initial stress and magnetic fields are absent, (47) produces the thermo-visco-elastic Rayleigh wave-velocity equation including strain and stress rates as,

$$
\left(2-\frac{C^{L}}{M_{S}^{*}}\right)^{2}\left(\sigma_{1}^{2}+\sigma_{1} \sigma_{2}+\sigma_{2}^{2}-1+\frac{C^{L}}{M_{L}^{*}}\right)-4 \sigma_{1} \sigma_{2} \sigma_{3}\left(\sigma_{1}+\sigma_{2}\right)=0
$$


When we neglect the viscosity effect also, a similar result is obtained for the Rayleigh wave-velocity equation in a thermal field and the reduced equation is in accordance with the result obtained by Chadwick (1960) for $h=0$.

Now, we consider the particular case 2 with the assumption that the length of the waves is large compared to the thickness $2 H$ of the layer. For this criterion we may approximate the hyperbolic tangents to the first term of their expansion into series. So we get from (38)

$$
\frac{\zeta_{1}}{\zeta_{2}}=-\left[P+Q \frac{\zeta_{3}}{\zeta_{2}}\right] / L .
$$

Here again, if we withdraw the magnetic field, the thermal field, and the initial stress, proceeding exactly in the same manner as done earlier we find from (49) that the wave velocity $c(=\omega / \eta)$ becomes zero. Hence, to obtain classical results parallel to those of Rayleigh (1885) and Lamb (1916) in visco-elastic media with stress and strain rates, the hyperbolic tangents have to be approximated up to the second term in their expansions. Classical results, due to Rayleigh (1885) and Lamb (1916) in elastic media, immediately follow from this equation on omitting the term due to viscosity.

At the end of the discussion, we consider the case when the wavelength is very small compared to the thickness of the layer. Here (38) reduces to the same form of (47) which gives the velocity of Rayleigh surface waves in magneto-thermo-visco-elastic initially stressed conducting layer, whose boundary is thermally insulated and which involves strain and stress rates.

\section{References}

Acharya D P, Sengupta P R 1978a Magneto-thermo-elastic waves in an initially stressed conducting layer. Gerlands Beitr. Geophys. 87: 229-239

Acharya D P, Sengupta P R 1978b Magneto-thermo-elastic surface waves in initially stressed conducting media. Acta Geophys. Pol. 26: 299-311

Bakshi S K 1970 On the effect of magneto-elastic interaction on the cooling process of an infinite circular cylinder. Proc. Cambridge Philos. Soc. 67: 173-180

Banos A 1956 Normal modes characterizing magneto-elastic plane waves. J. Phys. Rev. 104: 300-305

Bullen K E 1963 An introduction to the theory of seismology (Cambridge: University Press)

Carslaw H S, Jaeger J C 1959 Conduction of heat in solids (Oxford: University Press)

Chadwick P 1957 Elastic wave propagation in a magnetic field. IX Congress Int. Mech. Appl. 7: $143-158$

Chadwick P 1960 Progress in solid mechanics (eds) I N Sneddan, R Hill (Amsterdam: NorthHolland) vol. 1, chap 6

Das T K, Sengupta P R 1990a Surface waves in general visco-elastic media of higher order. Indian J. Pure Appl. Math. 21: 661-675

Das T K, Sengupta P R 1990b Surface waves in thermo-visco-elastic media considering time rate of stress and strain of higher order. Gerlands Beitr. Geophys. 99: 337-348

Das S C, Acharya D P, Sengupta P R 1994a Magneto visco-elastic surface waves in stressed conducting media. Sādhanā 19: 337-346

Das S C, Sengupta S, Acharya D P 1994b Magneto visco-elastic waves in an initially stressed conducting layer including strain rate and stress rate. Bull. Tech. Univ. Istanbul 47: 243-255 
De S N, Sengupta P R 1971 Surface waves in magneto-elastic initially stressed conducting media. Pure Appl. Geophys. 88: 44-52

De S N, Sengupta P R 1972 Magneto-elastic waves and disturbances in initially stressed conducting media. Pure Appl. Geophys. 93: 41-54

Ewing W M, Jardetsky W S, Press F 1957 Elastic waves in layered media (New York: McGrawHill) pp 257-259, 311

Flugge W 1967 Visco-elasticity (London: Blaisdel)

Knopoff $\mathrm{L} 1955$ The interaction between elastic wave motions and a magnetic field in elastic conductors. J. Geophys. Res. 60: 441-456

Lamb H 1916 On waves in elastic plate. Proc. R. Soc. (London) A93: 114-128

Nowacki W 1962 Thermo-elasticity (Oxford: Pergamon)

Pal K C, Sengupta P R 1987 Surface waves in visco-elastic solid of general type in the presence of thermal field and gravity. Proc. Indian Natl. Sci. Acad. A53: 353-372

Paria G 1962 On magneto-thermo-elastic plane waves. Proc. Cambridge Philos. Soc. 58: 527-531

Rayleigh Lord 1885 On waves propagated along the plane surface of an elastic solid. Proc. London Math. Soc. 17: 4-11

Roy S K, Sengupta P R 1985 Propagation of plane waves in a conducting thermo-visco-elastic solid of general nature placed in a magnetic field. Indian J. Theor. Phys. 33: 81-88

Willson W 1963 The propagation of magneto-thermo-elastic plane waves. Proc. Cambridge Philos. Soc. 59: 483-488

Yu C P, Tang S 1966 Magneto-elastic waves in initially stressed conductors. Z. Angew. Math. Phys. 17: 766-775 